\title{
Weak lensing evidence for a filament between A222/A223 †
}

\author{
Jörg P. Dietrich ${ }^{1}$, Douglas Clowe ${ }^{1}$, Peter Schneider ${ }^{1}$, Jürgen Kerp ${ }^{2}$ \\ and Emilio Romano-Díaz ${ }^{3}$ \\ ${ }^{1}$ IAEF, University of Bonn, Auf dem Hügel 71, 53121 Bonn, Germany email: \\ dietrich,clowe,peter@astro.uni-bonn.de \\ ${ }^{2}$ Radioastronomisches Institut, University of Bonn, Auf dem Hügel 71, 53121 Bonn, Germany \\ email: jkerp@astro.uni-bonn.de \\ ${ }^{3}$ Kapteyn Institute, University of Groningen, P.O. Box 800, 9700, Av. Groningen, The \\ Netherlands email: eromano@astro.rug.nl
}

\begin{abstract}
We present a weak lensing analysis and comparison to optical and X-ray maps of the close pair of massive clusters A222/223. Indications for a filamentary connection between the clusters are found and discussed.
\end{abstract}

\section{Introduction}

$N$-body simulations of cosmic structure formation predicts that matter in the universe should be concentrated along sheets and filaments and that clusters of galaxies form where these intersect (Kauffmann et al. 1999, Bond et al. 1996). This filamentary structure, often also dubbed "cosmic web", has been seen in galaxy redshift surveys (Vogeley et al. 1994) and X-rays (Zappacosta et al. 2002, Tittley \& Henriksen 2001).

Because of the greatly varying mass-to-light $(M / L)$ ratios between rich clusters and groups of galaxies (Tully \& Shaya 1998) it is problematic to convert the measured galaxy densities to mass densities without further assumptions. Weak gravitational lensing, which is based on the measurement of shape and orientation parameters of faint background galaxies (FBG), is a model-independent method to determine the surface mass density of clusters and filaments. Due to the finite ellipticities of the unlensed FBG, every weak lensing mass reconstruction is unfortunately an inherently noisy process, and the expected surface mass density of a single filament is too low to be detected with current telescopes (Jain et al. 2000).

Cosmic web theory also predicts that the surface mass density of a filament increases towards clusters (Bond et al. 1996). Filaments connecting neighboring clusters should have surface mass densities high enough to be detectable with weak lensing (Pogosyan et al. 1998). Such filaments may have been detected in several recent weak lensing studies.

Kaiser et al. (1998) found a possible filament between two of the three clusters in the $z=0.42$ super-cluster MS0302+17, but the detection remains somewhat uncertain because of a possible foreground structure overlapping the filament and possible edge effects due to the gap between two of the camera chips lying along the filament. Also, Gavazzi et al. (2004) could not confirm the detection of a filament in this system. Gray et al. (2002) claim to have found a filament extending between two of the three clusters of the Abell 901/902 super-cluster, but the significance of this detection is low and subject

$\dagger$ Based on observations made at ESO/La Silla under program Nos. 064.L-0248, 064.O-0248, 66.A-0165, 68.A-0269. 
to possible edge effects, as again the filament is close to the intersection of four chips of the camera.

\section{Data of the Abell 222/223 system}

A222/223 are two Abell clusters at $z \approx 0.21$ separated by $\sim 14^{\prime}$ on the sky, or $\sim$ $2600 h_{70}^{-1} \mathrm{kpc}$, belonging to the Butcher et al. (1983) photometric sample. Both clusters are rich, having Abell richness class 3 (Abell 1958). While these are optically selected clusters, they have been observed by ROSAT (Wang \& Ulmer 1997) and are confirmed to be massive clusters. Proust et al. (2000, P00) published a list of 53 spectra in the field of A222/223, 4 of them in region between the clusters (hereafter "intercluster region") and at the redshift of the clusters. Later, Dietrich et al. (2002, D02) reported on spectroscopy of 183 objects in the cluster field, 153 being members of the clusters or at the cluster redshift in the intercluster region. Taking the data of P00 and D02 together, 6 galaxies at the cluster redshift are known in the intercluster region, establishing this cluster system as a good candidate for a filamentary connection.

Imaging was performed with the Wide Field Imager (WFI) at the ESO/MPG $2.2 \mathrm{~m}$ telescope. In total, twenty $600 \mathrm{~s}$ exposures were obtained in $R$-band in October 2001 centered on A223, eleven $900 \mathrm{~s} R$-band exposures were taken in December 1999 centered on A222. The images were taken with a dithering pattern filling the gaps between the chips in the co-added images of each field.

The $R$-band data used for the weak lensing analysis is supplemented with three $900 \mathrm{~s}$ exposures in the $B$ and $V$-band centered on each cluster taken from November 1999 to December 2000. The final $B$ - and $V$-band images have some remaining gaps and regions that are covered by only one exposure and - due to the dithering pattern - do not cover exactly the same region as the $R$-band images.

\subsection{Catalog creation}

Lensing catalogs were created from the $R$-band images using the KSB algorithm (Kaiser et al. 1995, corrections in Hoekstra et al. 1998). Stellar reflection rings and diffraction spikes were masked and the masked regions excluded from the catalogs. The catalogs from both pointings were merged and the ellipticities of objects contained in both catalogs were averaged.

From this merged catalog all objects with $R<22 \mathrm{mag}$, signal-to-noise $\nu<7$, Gaussian radius 0 !' $4<r_{\mathrm{g}}<1^{\prime \prime} .2$, and ellipticity $e>0.8$ were deleted. All surviving objects fainter than $R>23.5$ mag were kept as likely background galaxies, while objects brighter than this - for which colors were available - were selected according to the following criteria: Objects detected in $B, V$, and $R$ matching colors of galaxies at $z<0.5,-0.23<$ $(V-R)-0.56 \times(B-V)<0.67,0.5<B-V<1.6$, were deleted from the sample. Galaxies detected only in the $V$ and $R$ band were kept if $(V-R)>1.0$. The final catalog contains 25583 objects, corresponding to a galaxy number density of $15.5 \mathrm{arcmin}^{-2}$ if the masked regions are not taken into account for the computation of the total area.

\section{Mass and light maps}

Fig. 1 shows a reconstruction obtained from the catalog described in the previous section. The reconstruction was performed on a $214 \times 200$ points grid using the algorithm of Seitz \& Schneider (2001) adapted to the field geometry. The smoothing scale of the shear data was set to $2^{\prime}$. Galaxies in the catalog were weighted by the error estimate of their initial ellipticity measurement. 

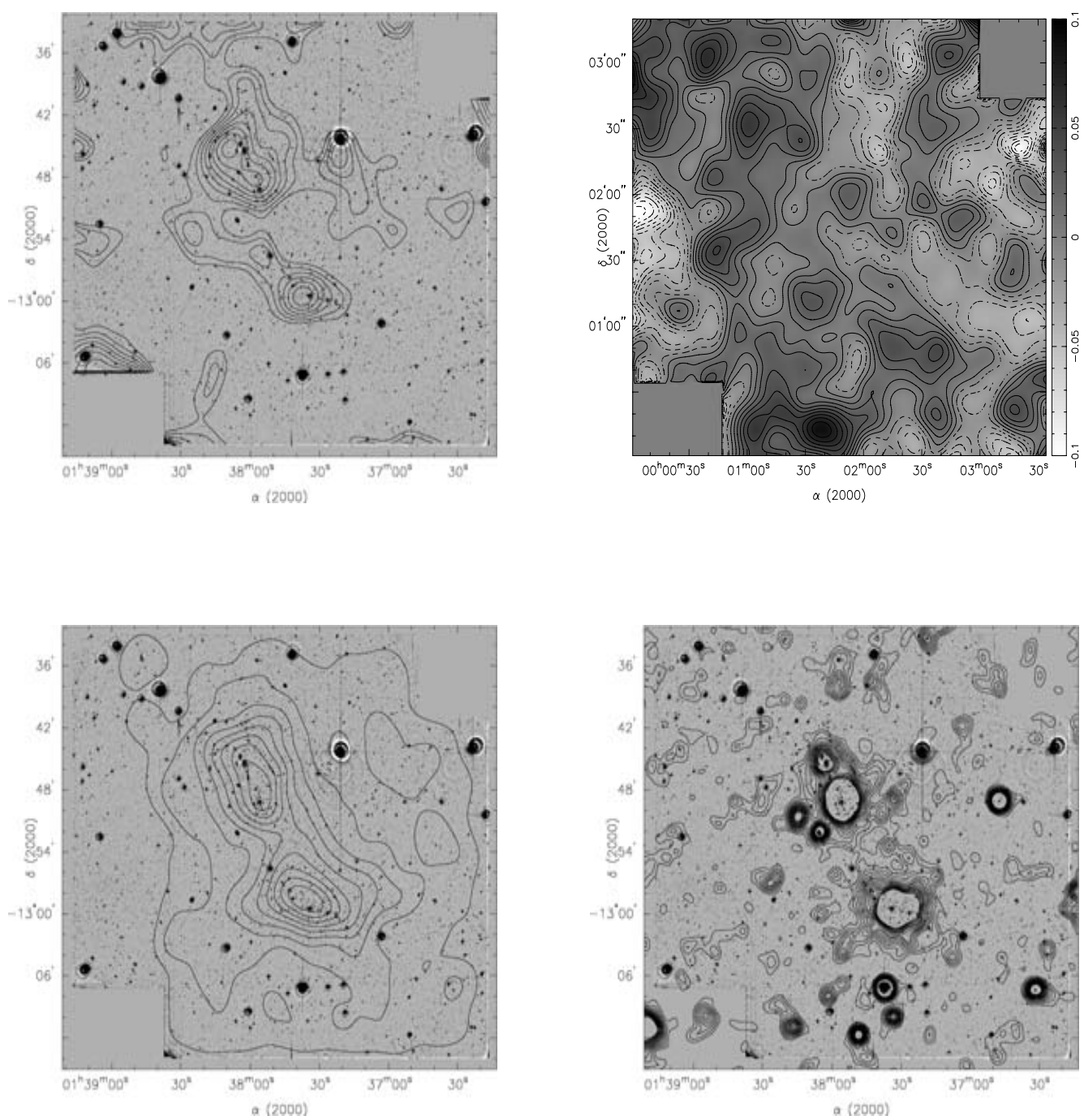

Figure 1. Various light and mass maps of the A222/223 system. A223 is the Northern cluster, A222 is South of it. Top left: Weak lensing reconstruction of A222/223. Each contour represents an increase in $\kappa$ of $0.01\left(\sim 3.2 \times 10^{13} h_{70} M_{\odot} \mathrm{Mpc}^{-2}\right.$, assuming $\left.\bar{z}_{\mathrm{FBG}}=1\right)$ above the mean $\kappa$ at the edge of the field. The peak $\sim 12^{\prime}$ SE of A222 has an optical counter-part. Top right: Surface mass density map created from a catalog with randomly rotated galaxies used to estimate the noise level of the reconstruction on the right. Bottom left: Density distribution of galaxies matching the colors of the red cluster sequence $(0.78<V-R<0.98)$ of the Abell clusters smoothed with a $2^{\prime}$ Gaussian. The absence of galaxies in the Eastern part is caused by the very bad or missing $V$-band coverage. Bottom right: Contours in this plot are from ROSAT PSPC data in the energy range $0.5-2.4 \mathrm{keV}$. The lowest contour is at the $6 \sigma$ level. Higher contours rise in steps of $1 \sigma$. Note that the extended X-ray emission North of A223 is associated with the Northern extension of A223 in the surface mass density map.

Both clusters are well detected in the reconstruction. The two components of A223 are clearly visible. The strong mass peak West of A223 is most likely associated with the reflection ring around the bright $V=7.98 \mathrm{mag}$ star at that position. Although the reflection ring and a large area around it were masked, diffuse stray light is visible 
extending beyond the masked region, well into A223, probably being the cause of the observed mass peak.

Also visible is a bridge in the surface mass density extending between A222 and A223. Although the signal of this possible filamentary connection between the clusters is very low, the feature is quite robust when the selection criteria of the catalog are varied and it never disappears.

The noise level in the reconstruction can be estimated by randomly rotating the galaxies while keeping their positions and ellipticity moduli fixed. Performing a reconstruction on this randomized catalog gives $\left\langle\kappa^{2}\right\rangle \simeq 0.015$, suggesting that the intercluster connection is present at the $\sim 2 \sigma$ level.

The galaxy density distribution and the X-ray contours in Fig. 1 both show a clear connection between the clusters. While the X-ray and galaxy density contours are aligned in the intercluster region, the surface mass density contours connect the clusters Eastwards of them.

\section{Discussion}

We presented weak lensing, optical galaxy density, and X-ray maps of the massive, close pair of galaxy clusters A222/223. All maps show a connection between the clusters in the intercluster region. The density distribution of color-selected objects, supplemented by the spectroscopic confirmation of galaxies at the cluster redshift in the intercluster region by P00 and D02, and the significant connection between both clusters in the $0.5-2.4 \mathrm{keV}$ band of ROSAT's PSPC establish a secure filamentary connection between the clusters. The weak lensing indications for a dark matter bridge are much less secure. The signalto-noise of the structure extending between the clusters is low but the structure itself is very robust and never disappears when the selection criteria for the lensing catalog are varied.

We note that the luminosity density of color-selected galaxies in the intercluster region is only by a factor of $\sim 2$ lower than in the peaks of the galaxy clusters. If the early type galaxies trace the mass well and a constant $M / L$ ratio is assumed, we would expect a clearly detectable lensing signal along the galaxy density contours. This is obviously not the case. A more detailled comparison of the observed and expected surface mass density, also addressing the apparent misalignment of mass and light in A223, will be done elsewhere (Dietrich et al. 2004, in preparation). We also note that a misalignment of mass and light is present in the filament candidate of Gray et al. (2002).

\section{Acknowledgements}

This work has been supported by the German Ministry for Science and Education (BMBF) through DESY under the project 05AE2PDA/8, and by the Deutsche Forschungsgemeinschaft under the project SCHN 342/3-1.

\section{References}

Abell, G. O. 1958, ApJS 3, 211

Bond, H., Kofman, L., \& Pogosyan, D. 1996, Nature 380, 603

Butcher, H., Wells, D. C., \& Oemler, A. 1983, ApJS 52, 183

David, L. P., Forman, W., \& Jones, C. 1999, ApJ 519, 533

Dietrich, J. P., Clowe, D. I., \& Soucail, G. 2002, A\&A 394, 395

Gavazzi, R., Mellier, Y., Fort, B., Cuillandre, J. \& Dantel-Fort, M. 2004, astro-ph/0401403

Gray, M. E., Taylor, A. N., Meisenheimer, K., et al. 2002, ApJ 568, 141 
Hoekstra, H., Franx, M., Kuijken, K., \& Squires, G. 1998, ApJ 504, 636

Jain, B., Seljak, U., \& White, S. 2000, ApJ 530, 547

Kaiser, N., Squires, G., \& Broadhurst, T. 1995, ApJ 449, 460

Kaiser, N., Wilson, G., Luppino, G., et al. 1998, astro-ph/99809268

Kauffmann, G., Colberg, J. M., Diaferio, A., \& White, S. D. M. 1999, MNRAS 303, 188

Pogosyan, D., Bond, J. R., Kofman, L., \& Wadsley, J. 1998, in Wide Field Surveys in Cosmology, 14th IAP meeting held May 26-30, 1998, Paris. Publisher: Editions Frontieres. ISBN: 28 6332-241-9, 61

Proust, D., Cuevas, H., Capelato, H. V., et al. 2000, A\&A 355, 443

Seitz, S. \& Schneider, P. 2001, $A \& A$ 374, 740

Tittley, E. R. \& Henriksen, M. 2001, ApJ 563, 673

Tully, R. B. \& Shaya, E. J. 1998, astro-ph/9810298

Vogeley, M. S., Park, C., Geller, M. J., Huchra, J. P., \& Gott, J. R. I. 1994, ApJ 420, 525

Wang, Q. D. \& Ulmer, M. P. 1997, MNRAS 292, 920

Zappacosta, L., Mannucci, F., Maiolino, R., et al. 2002, A\&A 394, 7 